\title{
Light Quark Masses 99 *
}

\author{
Stephan Narison ${ }^{\mathrm{a}}$ \\ ${ }^{a}$ Laboratoire de Physique Mathématique, Université de Montpellier II Place Eugène Bataillon, 34095 - \\ Montpellier Cedex 05, France and KEK Tsukuba, Ibaraki, 350801 - Japan. \\ E-mail: narison@lpm.univ-montp2.fr
}

I give a short historical and a critical review of the determinations of light quark masses from QCD at dawn of the next millennium. QCD spectral sum rules combined with ChPT give, to order $\alpha_{s}^{3}$, the world average for the running masses: $\bar{m}_{s}(2 \mathrm{GeV})=(118.9 \pm 12.2) \mathrm{MeV}, \bar{m}_{d}(2 \mathrm{GeV})=(6.3 \pm 0.8) \mathrm{MeV}, \bar{m}_{u}(2 \mathrm{GeV})=(3.5 \pm 0.4) \mathrm{MeV}$ and the corresponding values of the invariant masses given in Eq. 24. Lower and upper bounds derived from the positivity of spectral moments are presented in Tables 2 and 3. For a comparison, we critically review the recent lattice results (section 8 and Table 5) and attempt to deduce the present $Q C D$ grand average determination (Table 6$): \bar{m}_{s}(2$ $\mathrm{GeV})=(110.9 \pm 8.8) \mathrm{MeV}$, to be used with a great care. Then, we deduce the value: $B_{6}^{1 / 2}-0.45($ resp. 0.32$) \mathrm{B}_{8}^{3 / 2} \simeq$ $1.6 \pm 0.4$ (resp. $1.1 \pm 0.3$ ) and the lower bound $1.1 \pm 0.2$ (resp. $0.7 \pm 0.1$ ), for the combination of the penguin operators, governing the CP-violating parameters $\epsilon^{\prime} / \epsilon$ without (resp. with) the inclusion of the final state interaction effects. The result signals a possible deviation from the leading $1 / N$ prediction by about $1 \sim 3 \sigma$, which should be tested using accurate non-perturbative calculations.

\section{INTRODUCTION}

One of the most important parameters of the standard model and chiral symmetry is the light quark masses. Indeed, they are useful for a much better understanding of the realizations of chiral symmetry breaking [1] 3] and for some eventual explanation of the origin of quark masses in unified models of interactions 仰. Within some popular parametrizations of the hadronic matrix elements [5], the strange quark mass can also largely influence the Standard Model prediction of the $C P$ violating parameters $\epsilon^{\prime} / \epsilon$, which have been mesured recently [6]. However, contrary to the leptons, where the physical masses can be identified with the pole of the propagator 2 , the quark masses are difficult to define because of confinement. Instead, they can be treated as coupling constants of the QCD Lagrangian, where the notion of the running and invariant masses, which

\footnotetext{
* Review talk given at the QCD 99 Euroconference (Montpellier 7-13th July 1999) and plenary talk given at the QCD Confinement 2000 (Osaka 7-10th March 2000).

${ }^{2}$ For a first explicit definition of the perturbative quark pole mass in the $\overline{M S}$-scheme, see 7 (renormalizationscheme invariance) and (8) (regularization-scheme invariance).
}

are renormalization scheme and scale dependents, has been introduced [9]. In practice, these masses are conveniently defined within the standard $\overline{M S}$ scheme. In addition to the determination of the ratios of light quark masses (which are scale independent) from current algebra [1], and from chiral perturbation theory (ChPT), its modern version [3], a lot of effort reflected in the literature 10 has been put into extracting directly from the data the running quark masses using the SVZ 11] QCD spectral sum rules (QSSR) [12], LEP experiments [14] and lattice data 13]. In this talk, I shall review the different determinations from these QCD approaches, by emphasizing the historical developments of the field.

\section{RUNNING AND INVARIANT LIGHT QUARK MASSES IN QCD}

It is convenient to introduce the dimensionless coupling $x_{i}(\nu) \equiv m_{i}(\nu) / \nu$, where $\nu$ is the renormalization scheme subtraction constant. The running quark mass is a solution of the differential equation:

$\frac{d \bar{x}_{i}}{d t}=\left(1+\gamma\left(\alpha_{s}\right)\right) \bar{x}_{i}(t): \bar{x}_{i}(t=0)=x_{i}(\nu)$. 
In the $\overline{M S}$-scheme, its solution to order $a_{s}^{3}\left(a_{s} \equiv\right.$ $\left.\alpha_{s} / \pi\right)$ is:

$$
\begin{aligned}
\bar{m}_{i}(\nu) & =\hat{m}_{i}\left(-\beta_{1} a_{s}(\nu)\right)^{-\gamma_{1} / \beta_{1}}\{1 \\
& +\frac{\beta_{2}}{\beta_{1}}\left(\frac{\gamma_{1}}{\beta_{1}}-\frac{\gamma_{2}}{\beta_{2}}\right) a_{s}(\nu) \\
& +\frac{1}{2}\left[\frac{\beta_{2}^{2}}{\beta_{1}^{2}}\left(\frac{\gamma_{1}}{\beta_{1}}-\frac{\gamma_{2}}{\beta_{2}}\right)^{2}-\frac{\beta_{2}^{2}}{\beta_{1}^{2}}\left(\frac{\gamma_{1}}{\beta_{1}}-\frac{\gamma_{2}}{\beta_{2}}\right)\right. \\
& \left.+\frac{\beta_{3}}{\beta_{1}}\left(\frac{\gamma_{1}}{\beta_{1}}-\frac{\gamma_{3}}{\beta_{3}}\right)\right] a_{s}^{2}(\nu) \\
& \left.+1.95168 a_{s}^{3}\right\},
\end{aligned}
$$

where the $a_{s}^{3}$ term comes from [15]; $\gamma_{i}$ are the $\mathcal{O}\left(a_{s}^{i}\right)$ coefficients of the quark-mass anomalous dimension, which read for three flavours:

$\gamma_{1}=2, \quad \gamma_{2}=91 / 12, \quad \gamma_{3}=24.8404$

The invariant mass $\hat{m}_{i}$ has been introduced for the first time by [9] in connection with the analysis of the breaking of the Weinberg sum rules by the quark mass terms in QCD.

\section{RATIOS OF LIGHT QUARK MASSES}

The ratios of light quark masses are welldetermined from current algebra [1], and ChPT [3]. In this approach, the meson masses are expressed using a systematic expansion in terms of the light quark masses:

$$
\begin{aligned}
M_{\pi^{+}}^{2} & =\left(m_{u}+m_{d}\right) B+\mathcal{O}\left(m^{2}\right)+\ldots \\
M_{K^{+}}^{2} & =\left(m_{u}+m_{s}\right) B+\mathcal{O}\left(m^{2}\right)+\ldots \\
M_{K^{0}}^{2} & =\left(m_{d}+m_{s}\right) B+\mathcal{O}\left(m^{2}\right)+\ldots
\end{aligned}
$$

where $B \equiv-\langle\bar{\psi} \psi\rangle / f_{K}^{2}$ from the Gell-Mann, Oakes, Renner relation [16]:

$m_{\pi}^{2} f_{\pi}^{2} \simeq-\left(m_{u}+m_{d}\right)\langle\bar{\psi} \psi\rangle+\mathcal{O}\left(m^{2}\right)$.

However, only the ratio, which is scale independent can be well determined. To leading order in

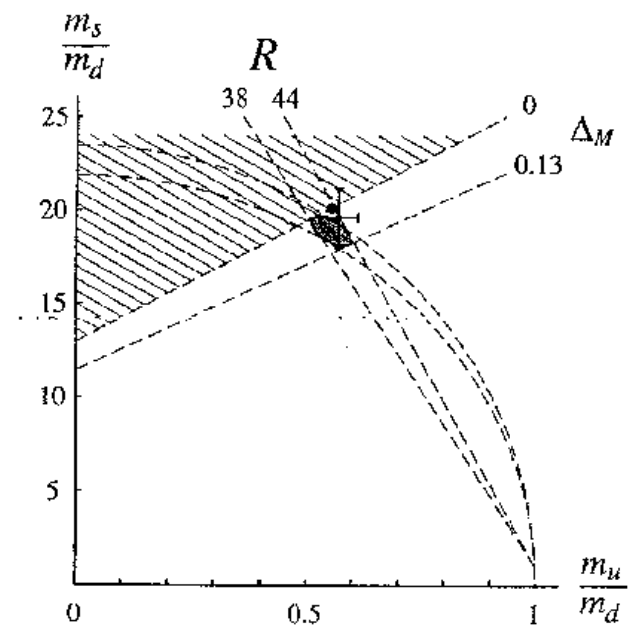

Figure 1. $m_{s} / m_{d}$ versus $m_{u} / m_{d}$ from [17].

$m$ 17]:

$$
\begin{aligned}
& \frac{m_{u}}{m_{d}} \approx \frac{M_{\pi^{+}}^{2}-M_{K^{0}}^{2}+M_{K^{+}}^{2}}{M_{\pi^{+}}^{2}+M_{K^{0}}^{2}-M_{K^{+}}^{2}} \approx 0.66 \\
& \frac{m_{s}}{m_{d}} \approx \frac{-M_{\pi^{+}}^{2}+M_{K^{0}}^{2}+M_{K^{+}}^{2}}{M_{\pi^{+}}^{2}+M_{K^{0}}^{2}-M_{K^{+}}^{2}} \approx 20
\end{aligned}
$$

Including the next order + electromagnetic corrections, the ratios of masses are constrained on the ellipse:

$$
\left(\frac{m_{u}}{m_{d}}\right)^{2}+\frac{1}{Q^{2}}\left(\frac{m_{s}}{m_{d}}\right)^{2}=1
$$

where: $Q^{2} \simeq\left(m_{s}^{2}-\hat{m}^{2}\right) /\left(m_{d}^{2}-m_{u}^{2}\right)=22.7 \pm 0.8$ using the value of the $\eta \rightarrow \pi^{+} \pi^{-} \pi^{0}$ from the PDG average [10], though this value can well be in the range 22-26, to be compared with the Dashen's formula 19] of $24.2 ; \hat{m} \equiv(1 / 2)\left(m_{u}+m_{d}\right)$. In Fig. 1 , one shows the range spanned by $R \equiv\left(m_{s}-\right.$ $\hat{m}) /\left(m_{d}-m_{u}\right)$ and the corrections to the GMO mass formula $\Delta_{M}: M_{8}^{2}=(1 / 3)\left(4 M_{K}^{2}-m_{\pi}^{2}\right)(1+$ $\left.\Delta_{M}\right)$. The Weinberg mass ratio [1] is also shown

${ }^{3}$ In Generalized ChPT, the contribution of the $m^{2}$-term can be as large as the $m$ one 18, which modifies drastically these ratios. 
which corresponds to the Dashen's formula and $R \simeq 43$. At the intersection of different ranges, one deduces [17]:

$$
\begin{aligned}
& \frac{m_{u}}{m_{d}}=0.553 \pm 0.043, \frac{m_{s}}{m_{d}}=18.9 \pm 0.8, \\
& \frac{2 m_{s}}{\left(m_{d}+m_{u}\right)}=24.4 \pm 1.5 .
\end{aligned}
$$

The possibility to have a $m_{u}=0$ advocated in 20 appears to be unlikely as it implies too strong flavour symmetry breaking and is not supported by the QSSR results from 2-point correlators of the divergences of the axial and vector currents, as will be shown in the next sections.

\section{QCD SPECTRAL SUM RULES}

\subsection{Description of the method}

Since its discovery in 79 [11], QSSR has proved to be a powerful method for understanding the hadronic properties in terms of the fundamental QCD parameters such as the QCD coupling $\alpha_{s}$, the (running) quark masses and the quark and/or gluon $\mathrm{QCD}$ vacuum condensates. The description of the method has been often discussed in the literature, where a pedagogical introduction can be, for instance, found in the book [12]. In practice (like also the lattice), one starts the analysis from the two-point correlator:

$\psi_{H}\left(q^{2}\right) \equiv i \int d^{4} x e^{i q x}\left\langle 0\left|\mathcal{T} J_{H}(x)\left(J_{H}(0)\right)^{\dagger}\right| 0\right\rangle$,

built from the hadronic local currents $J_{H}(x)$, which select some specific quantum numbers. However, unlike the lattice which evaluates the correlator in the Minkowski space-time, one exploits, in the sum rule approaches, the analyticity property of the correlator which obeys the well-known Källen-Lehmann dispersion relation:

$\psi_{H}\left(q^{2}\right)=\int_{0}^{\infty} \frac{d t}{t-q^{2}-i \epsilon} \frac{1}{\pi} \operatorname{Im} \psi_{H}(t)+\ldots$,

where ... represent subtraction points, which are polynomials in the $q^{2}$-variable. In this way, the sum rule expresses in a clear way the duality between the integral involving the spectral function $\operatorname{Im} \psi_{H}(t)$ (which can be measured experimentally), and the full correlator $\psi_{H}\left(q^{2}\right)$. The latter can be calculated directly in the QCD Euclidean space-time using perturbation theory (provided that $-q^{2}+m^{2}$ ( $m$ being the quark mass) is much greater than $\Lambda^{2}$ ), and the Wilson expansion in terms of the increasing dimensions of the quark and/or gluon condensates which simulate the non-perturbative effects of QCD.

\subsection{Beyond the usual SVZ expansion}

Using the Operator Product Expansion (OPE) [11], the two-point correlator reads:

$\psi_{H}\left(q^{2}\right) \simeq \sum_{D=0,2, \ldots} \frac{1}{\left(q^{2}\right)^{D / 2}} \sum_{\operatorname{dim} O=D} C\left(q^{2}, \nu\right)\langle\mathcal{O}(\nu)\rangle$,

where $\nu$ is an arbitrary scale that separates the long- and short-distance dynamics; $C$ are the Wilson coefficients calculable in perturbative QCD by means of Feynman diagrams techniques; $\langle\mathcal{O}(\nu)\rangle$ are the quark and/or gluon condensates of dimension $D$. In the massless quark limit, one may expect the absence of the terms of dimension 2 due to gauge invariance. However, it has been emphasized recently [21] that the resummation of the large order terms of the perturbative series, and the effects of the higher dimension condensates due e.g. to instantons, can be mimiced by the effect of a tachyonic gluon mass $\lambda$ which generates an extra $D=2$ term not present in the original OPE. Its presence might be understood from the analogy with the short distance linear part of the QCD potential f. The strength of this short distance mass has been estimated from the $e^{+} e^{-}$data to be [23,24]:

$\frac{\alpha_{s}}{\pi} \lambda^{2} \simeq-(0.06 \sim 0.07) \mathrm{GeV}^{2}$,

which leads to the value of the square of the (short distance) string tension: $\sigma \simeq-\frac{2}{3} \alpha_{s} \lambda^{2} \simeq$ $[(400 \pm 20) \mathrm{MeV}]^{2}$ in an (unexpected) good agreement with the lattice result [25] of about [(440土 38) $\mathrm{MeV}]^{2}$. In addition to Eq. 5, the strengths of the vacuum condensates having dimensions $D \leq 6$ are also under good control, namely:

- $\langle\bar{s} s\rangle /\langle\bar{d} d\rangle \simeq 0.7 \pm 0.2$ from the meson 12 and baryon systems [26];

\footnotetext{
${ }^{4}$ Some evidence of this term is found from the lattice analysis of the static quark potential [22], though the extraction of the continuum result needs to be clarified.
} 
- $\left\langle\alpha_{s} G^{2}\right\rangle \simeq(0.07 \pm 0.01) \mathrm{GeV}^{4}$ from sum rules of $e^{+} e^{-} \rightarrow \mathrm{I}=1$ hadrons [23] and heavy quarkonia [27 29], and from the lattice 30;

- $g\left\langle\bar{\psi} \lambda_{a} / 2 \sigma^{\mu \nu} G_{\mu \nu}^{a} \psi\right\rangle \simeq(0.8 \pm 0.1) \mathrm{GeV}^{2}\langle\bar{\psi} \psi\rangle$, from the baryons [31,26] and the heavy-light mesons 32];

- $\alpha_{s}\langle\bar{u} u\rangle^{2} \simeq 5.8 \times 10^{-4} \mathrm{GeV}^{6}$ from $e^{+} e^{-} \rightarrow$ $I=1$ hadrons 23 ;

- $g^{3}\left\langle G^{3}\right\rangle \approx 1.2 \mathrm{GeV}^{2}\left\langle\alpha_{\mathrm{s}} \mathrm{G}^{2}\right\rangle$ from dilute gaz instantons 33].

\subsection{Spectral function}

In the absence of the complete data, the spectral function is often parametrized using the "naïve" duality ansatz:

$$
\begin{aligned}
& \frac{1}{\pi} \operatorname{Im} \psi_{H}(t) \simeq 2 M_{H}^{2 n} f_{H}^{2} \delta\left(t-M_{H}^{2}\right)+ \\
& \text { "QCD continuum" } \times \theta\left(\mathrm{t}-\mathrm{t}_{\mathrm{c}}\right),
\end{aligned}
$$

which has been tested 12 using $e^{+} e^{-}$and $\tau$ decay data, to give a good description of the spectral integral in the sum rule analysis; $f_{H}$ (analogue to $f_{\pi}$ ) is the hadron's coupling to the current ; $2 n$ is the dimension of the correlator; while $t_{c}$ is the QCD continuum's threshold.

\subsection{Form of the sum rules and optimiza- tion procedure}

Among the different sum rules discussed in the literature [12], we shall be concerned with the:

- Laplace sum rule (LSR) [11,34,28:

$\mathcal{L}_{n}(\tau)=\int_{0}^{\infty} d t t^{n} \exp (-t \tau) \frac{1}{\pi} \operatorname{Im} \psi_{H}(t)$.

The advantage of the Laplace sum rules with respect to the previous dispersion relation is the presence of the exponential weight factor, which enhances the contribution of the lowest resonance and low-energy region accessible experimentally. For the QCD side, this procedure has eliminated the ambiguity carried by subtraction constants, arbitrary polynomial in $q^{2}$, and has improved the convergence of the OPE by the presence of the factorial dumping factor for each condensates of given dimensions. As one can notice, there are "a priori" two free external parameters $\left(\tau, t_{c}\right)$ in the analysis. The optimized result will be (in principle) insensitive to their variations. In some cases, the $t_{c}$-stability is not reached due to the too naïve parametrization of the spectral function. One can either fix the $t_{c}$-values by the help of FESR (local duality) or improve the parametrization of the spectral function by introducing threshold effects fixed by chiral perturbation theory, ..., in order to restore the $t_{c}$-stability of the results. The results discussed below satisfy these stability criteria.

- $\tau$-like sum rules [35]-38]:

$\mathcal{R}_{n}^{m}=\int_{0}^{M_{\tau}^{2}} d t t^{n}\left(1-\frac{t}{M_{\tau}}\right)^{m} \frac{1}{\pi} \operatorname{Im} \psi_{H}(t)$,

The advantage of the $\tau$-like sum rule is the presence of the threshold factor which gives a zero near the real axis where QCD is not expected to be applicable. Optimal results should be insensitive to the changes of $M_{\tau}$, and to the values of the degrees $(m, n)$ of the moments.

- Finite Energy Sum Rule (FESR) [39]-[41]:

$\mathcal{M}_{n}\left(t_{c}\right)=\int_{0}^{t_{c}} d t t^{n} \frac{1}{\pi} \operatorname{Im} \psi_{H}(t)$,

The advantage of the FESR is the separation (to leading order in $\alpha_{s}$ ) of the terms of given dimensions, which gives a set of local duality constraints. However, unlike the two formers, FESR is sensitive to the high-energy tails of the spectral integral and needs an accurate treatment of this region, in order that the optimal results are insensitive to the changes of $t_{c}$.

\section{UP AND DOWN RUNNING MASSES}

\subsection{Pseudoscalar sum rules}

- Values of $\left(\bar{m}_{u}+\bar{m}_{d}\right)$ have been extracted for the first time in 42,34 using the sum rule of the 2-point correlator associated to the pseudoscalar current:

$\partial_{\mu} A^{\mu}(x)=\left(m_{i}+m_{j}\right): \bar{u}\left(i \gamma_{5}\right) d:$.

The analysis has been improved (or disproved) later on by many groups [2, 12], 433]-[50], by the inclusion of higher order terms or/and by a more involved parametrization of the spectral function (threshold effects, ChPT,...). However, this channel is quite peculiar due to the Goldstone nature 
Table 1

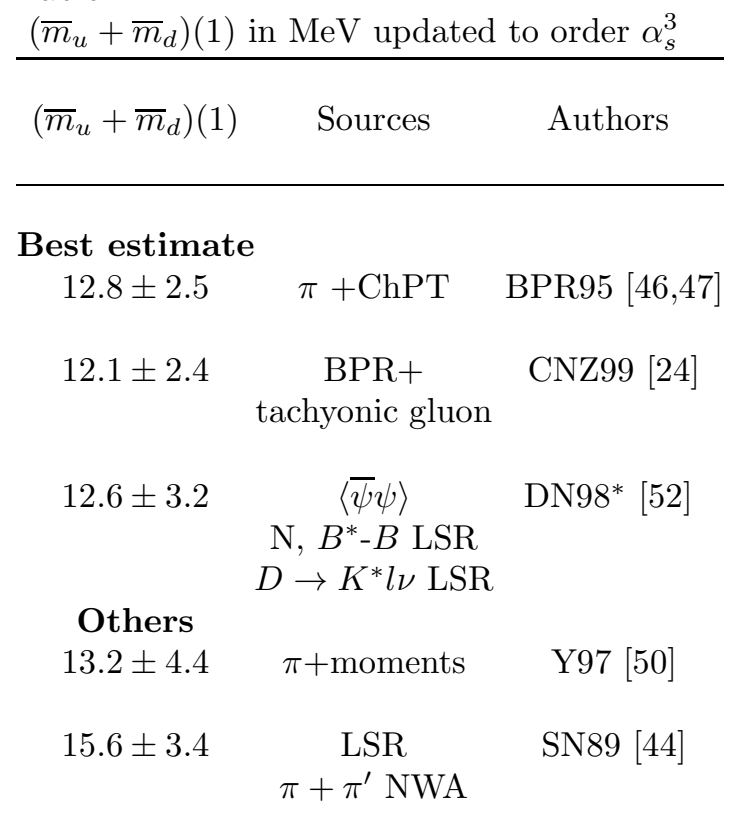

\section{$13.1 \pm 1.5($ stat $) \quad \pm 1.3($ syst $) \quad$ Average}

* to order $\alpha_{s}$ and not included in the average.

of the pion, where the value of the sum rule scale $\left(1 / \tau\right.$ for Laplace and $t_{c}$ for FESR $)$ is relatively large of about $2 \mathrm{GeV}^{2}$ compared with the pion mass, where the duality between QCD and the pion is lost. This implies an important role of the higher states (radial excitations or/and theoretical parametrizations of the spectral function above the $3 \pi$ threshold) in the analysis, and then led to some controversial results, which hopefully can be cured by the presence of the new $1 / q^{2}$ 21,24] due to the tachyonic gluon mass, which enlarges the duality region to lower scale and then minimizes the role of the higher states into the sum rule. The errors due to the QCD part of the sum rules, which is now known to order $\alpha_{s}^{3}$, are much less than from the parametrization of the spectral function. Among the available results, we consider that the best estimates of $\left(\bar{m}_{u}+\bar{m}_{d}\right)$ from this channel come from 46$](\pi+$ ChPT parametrization of the $3 \pi$ continuum) and
Table 2

Lower bounds on $\bar{m}_{u, d, s}(2)$ in $\mathrm{MeV}$ to order $\alpha_{s}$

\begin{tabular}{ccr}
\hline Observables & Sources & Authors \\
\hline $\bar{m}_{u}+\bar{m}_{d}$ & & \\
8 & $\pi, \sigma$ & LRT97 [49] \\
7.3 & $\pi$ & Y97[50] \\
7. & $\langle\bar{\psi} \psi\rangle+$ GMOR & DN98[52] \\
$\bar{m}_{d}-\bar{m}_{u}$ & & \\
1.5 & $K \pi$ & Y97[50] \\
$\bar{m}_{s}$ & & \\
100 & $K$ & LRT97[49] \\
104 & $K$ & Y97[50] \\
90 & $\langle\bar{\psi} \psi\rangle+\mathrm{ChPT}$ & DN98[52] \\
\hline
\end{tabular}

from 24 (inclusion of the tachyonic gluon mass into the analysis of [46]). The result of 46 to order $\alpha_{s}^{2}$ has been extended to order $\alpha_{s}^{3}$ by [47]. Also the result of [46] updates the one in 445. Ref. [50] uses the positivity of the higher state contributions plus the moment inequalities to order $\alpha_{s}$. In 44], one treats the $\pi^{\prime}$ in a Narrow Width Approximation (NWA), while the QCD expression is to order $\alpha_{s}^{2}$. These different determinations are quoted in Table 1 , after including into these published results the perturbative contributions of the known $\alpha_{s}^{3}$-term [51]. The effect of this term is quite small as the PT series converges quite well at the sum rule working region of about $1.5 \mathrm{GeV}$. Indeed, the PT expression of e.g. the Laplace sum rule normalized to $\left(\bar{m}_{u}+\bar{m}_{d}\right)^{2}$ reads:

$\mathcal{L} \sim 1+4.82 a_{s}+21.98 a_{s}^{2}+53.14 a_{s}^{3}+\mathcal{O}\left(a_{s}^{4}\right) .(17)$

One can notice the good consistency of the results from the different forms of the sum rules in the pion channel. We give in Table 1 the average of these updated determinations, where we have added an extra $10 \%$ error which takes into 
Table 3

Upper bounds on $\bar{m}_{u, d, s}(2)$ in $\mathrm{MeV}$ from 52] (resp. 65]) to order $\alpha_{s}$ (resp. $\alpha_{s}^{3}$ )

\begin{tabular}{ccc} 
Observables & Sources & Authors \\
\hline $\bar{m}_{u}+\bar{m}_{d}$ & & \\
11.5 & $\langle\bar{\psi} \psi\rangle+\mathrm{GMOR}$ & DN98 52 \\
$\bar{m}_{s}$ & & \\
148 & $\langle\bar{\psi} \psi\rangle+\mathrm{ChPT}$ & $\mathrm{DN} 9852$ \\
$147 \pm 21$ & $e^{+} e^{-}+\tau$-decay & SN99 65 \\
\hline
\end{tabular}

account the systematics of the approach?

- Lower bounds for $\left(\bar{m}_{u}+\bar{m}_{d}\right)$ based on moments inequalities and the positivity of the spectral functions have been obtained, for the first time, in 42, 34. These bounds have been rederived recently in 49,50 to order $\alpha_{s}$. Their optimal values quoted in Table 2 exclude the low value of about $6 \mathrm{MeV}$ given by 48 .

\subsection{Scalar sum rules}

- Lower bounds on $\left(\bar{m}_{d}-\bar{m}_{u}\right)$ have been extracted for the first time in 54 using the sum rule of the 2-point correlator associated to the scalar current:

$\partial_{\mu} V^{\mu}(x)=\left(m_{d}-m_{u}\right): \bar{d}(i) u:$,

which is sensitive to leading order to the quarkmass difference. The analysis has been extended later on by many authors [12,45,50]. However, the analysis relies heavily on the less controlled nature of the $a_{0}(980)$, where its $\bar{q} q$ nature appears to be favoured by the present data [55]. In the $I=$ 0 channel, the situation of the $\pi-\pi$ continuum is much more involved due to the possible gluonium nature of the low mass and wide $\sigma$ meson 56,57 ,

${ }^{5}$ The estimation of the systematic error is based on the (un)ability of the method for reproducing the hadron masses and couplings 11. 12 .

${ }^{6}$ The inclusion of the $\alpha_{s}^{3}$ term will decrease by about $10 \%$ the strength of these bounds, which is within the expected accuracy of the result.
55]. Instead, one can also use these sum rules the other way around, i.e. by using the values of the quark masses from the pseudoscalar sum rules and their ratio from $\mathrm{ChPT}$, in order to test the nature of these resonances [12,57.

5.3. Direct extraction of $\langle\bar{\psi} \psi\rangle$

- The chiral condensate can be directly extracted from the sum rules (nucleon, $B^{*}-B$ splitting, vector form factor of $D^{*} \rightarrow K^{*} l \nu$ ), which are particularly sensitive to it and to the mixed condensate $\left\langle\bar{\psi} \sigma^{\mu \nu}\left(\lambda_{a} / 2\right) G_{\mu \nu}^{a} \psi\right\rangle \equiv M_{0}^{2}\langle\bar{\psi} \psi\rangle$. A global fit from these different channels gives, to order $\alpha_{s}$, the running condensate value at $1 \mathrm{GeV}$ [52]:

$0.6 \leq\langle\bar{\psi} \psi\rangle /[-225 \mathrm{MeV}]^{3} \leq 1.5$,

a result also recovered by the lattice [53].

- Lower and upper bounds on the light quark masses given in Tables 2 and 3, can be obtained by transforming this result using the PCAC relation in Eq. 5, and the positivity of the $\mathcal{O}\left(\mathrm{m}^{2}\right)$ term. These results are independent on how chiral symmetry is realized (ChPT or generalized ChPT ?).

\section{4. $\bar{m}_{u, d, s}$ to order $\alpha_{s}^{3}$ from sum rules + ChPT}

One should note from Table 1 the consistency of the results from the pion channel and the one from the direct extraction of $\langle\bar{\psi} \psi\rangle$, which is an a posteriori support of the validity of the OPE for the $\pi$-sum rule in the working region, and signals the absence of the large effects due to instantons, which may break the OPE. Using the ratios from ChPT in section (2), one can deduce in units of $\mathrm{MeV}$, the value of the running masses at $2 \mathrm{GeV}$ to order $\alpha_{s}^{3}$ given in Table 6 . We have used the conversion factor:

$\bar{m}_{i}(1) \simeq(1.38 \pm 0.06) \bar{m}_{i}(2)$,

for running, to order $\alpha_{s}^{3}$, the results from 1 to $2 \mathrm{GeV}$, which corresponds to the average value of the QCD scale $\Lambda_{3} \simeq(375 \pm 50) \mathrm{MeV}$ from PDG [10] and others [58]. I remind that the errors in these determinations already take into account the systematics of the method (see Table 1).

\section{DIRECT EXTRACTIONS OF $\bar{m}_{s}$}


Table 4

Direct extractions of $\bar{m}_{s}(1)$ in $\mathrm{MeV}$ to order $\alpha_{s}^{3}$. Channels $\bar{m}_{s}(1)$ Sources Authors

$$
\begin{array}{cc}
\text { Kaon SR } & 165 \pm 15 \\
& 155 \pm 25 \\
& \mathbf{1 5 5} \pm \mathbf{2 5} \\
& \\
\text { Scalar SR } & 203 \pm 20 \\
143 \pm 17 \\
160 \pm 30 \\
159 \pm 11 \\
\mathbf{1 7 5} \pm \mathbf{4 8}
\end{array}
$$$$
\begin{array}{ccc}
(\tau \text {-like } \phi \text { SR } 173 \pm 33 & R_{\phi} \\
e^{+}-e^{-} \text {data } & 176 \pm 31 & \Delta_{10} \\
+\tau \text {-decay }) & 186 \pm 31 & \Delta_{1 \phi} \\
& \mathbf{1 7 9} \pm \mathbf{3 9} &
\end{array}
$$

$$
\begin{array}{cc}
\Delta S=-1 & 234_{-76}^{+61} \\
\text { part of } & 200 \pm 50 \\
\tau \text {-decay } & 164 \pm 33 \\
& \mathbf{2 1 3} \pm \mathbf{8 2}
\end{array}
$$

\section{SN89* 44 DPS99 "59 \\ Largest Range}

$$
\begin{gathered}
\text { CPS97 } 51 \\
\text { CFNP97 } 62 \\
\text { J98 63 } \\
\text { M99 64 } \\
\text { Largest Range }
\end{gathered}
$$

SN95,99 37,65

\section{Largest Range}

Aleph99

CKP98 [68]

PP99 70

\section{Average of Largest Ranges $166.7 \pm 18.8$}

\subsection{Pseudoscalar sum rules}

In the strange quark channel, we quote in Table 4 the results from [44] and [59], and consider the largest range spanned by these previously quoted results. We consider that this conservative range already takes into account and may even overestimate the systematics of the method. One should notice here that, unlike the case of the pion, the result is less sensitive to the contribution of the higher states continuum due to the relatively higher value of $M_{K}$, though the parametrization of the spectral function still gives larger errors than the QCD series.

\subsection{Scalar sum rules}

Following the pioneer's analysis of |54 , $m_{s}$ has been obtained by different authors 60 -64 by using the $K \pi$ phase shift data for parametrizing the spectral function. The different values obtained from this channel to order $\alpha_{s}^{3}$ is given in Table 4. Like in the case of the pseudoscalar channel, the errors are dominated by the uncertainties for parametrizing the spectral function.

\section{3. $m_{s}$ from $e^{+} e^{-}+\tau$-decay data}

One can combine the $e^{+} e^{-} \rightarrow I=0,1$ hadrons and the rotated recent $\Delta S=0$ component of the $\tau$-decay data in order to extract $m_{s}$. Unlike previous sum rules, one has the advantage to have a complete measurement of the spectral function in the region covered by the analysis. We shall work with:

$$
\begin{aligned}
R_{\tau, \phi} \equiv & \frac{3\left|V_{u d}\right|^{2}}{2 \pi \alpha^{2}} S_{E W} \int_{0}^{M_{\tau}^{2}} d s\left(1-\frac{s}{M_{\tau}^{2}}\right)^{2} \\
& \left(1+\frac{2 s}{M_{\tau}^{2}}\right) \frac{s}{M_{\tau}^{2}} \sigma_{e^{+} e^{-} \rightarrow \phi, \phi^{\prime}, \ldots},
\end{aligned}
$$

and the $S U(3)$-breaking combinations [37,65]:

$\Delta_{1 \phi} \equiv R_{\tau, 1}-R_{\tau, \phi}, \quad \Delta_{10} \equiv R_{\tau, 1}-3 R_{\tau, 0}$,

which vanish in the $S U(3)$ symmetry limit; $\Delta_{10}$ involves the difference of the isoscalar $\left(R_{\tau, 0}\right)$ and isovector $\left(R_{\tau, 1}\right)$ sum rules à la Das-mathurOkubo [66]. The PT series converges quite well at the optimization scale of about $1.6 \mathrm{GeV}$ 65]. E.g, normalized to $\bar{m}_{s}^{2}$, one has:

$\Delta_{1 \phi} \sim 1+\frac{13}{3} a_{s}+30.4 a_{s}^{2}+(173.4 \pm 109.2) a_{s}^{3} \cdot(23)$

It has been argued in [67] that $\Delta_{10}$ can be affected by large $S U(2)$ breakings, but this claim has not been confirmed from the result based on the other sum rules not affected by these terms [65]. The largest range of values from different form of the sum rules is given in Table 4, which one can compare with the average of $(178 \pm 33)$ $\mathrm{MeV}$ given in [65]. An upper bound deduced from the positivity of $R_{\tau, \phi}$ is given in Table 3 .

6.4. $m_{s}$ from the $\Delta S=-1$ part of $\tau$-decay One can also extract $m_{s}$ from the Cabibbo suppressed channel of $\tau$-decay [14,68 70], using different $\tau$-like moments. Unlike the case of the 
neutral $\phi$-meson current, where the QCD series is more convergent, here the convergence is quite bad, such that one needs to select an appropriate combination (spin $1+0$ pieces) for obtaining an acceptable result. Though a complete agreement has been obtained in the previous analysis of 69 with the two other determinations [14,68, a recent analysis in 70 is lower and more precise than the former, though still in agreement with the previous ones due to the generous errors given there. Ref. [70] argues that one should consider the previous results as an upper bound rather than a determination, an argument which needs to be confirmed. By inspecting the results in [70, we notice that the estimate decreases with increasing power of moments, rather than stabilizing. Therefore, it can be more appropriate to consider the conservative range spanned by the results from the three moments which is $(180 \pm 68)$ $\mathrm{MeV}$, rather than taking their average quoted in Table 4 from [70]. This conservative value is in better agreement with the two other determinations. It is also interesting to notice that the results from $\tau$-decay are in good agreement with the one from $e^{+} e^{-}$data, an agreement which is a priori expected because of the similarity of the two approaches.

6.5. $m_{s}$ and $m_{s} /\left(m_{u}+m_{d}\right)$ from QSSR

More generally, one can notice from Table 4 that there is a total agreement of the sum rule results from different channels. As already mentioned, we expect that the largest ranges given in Table 4 already include the QSSR systematics. Using the average of these largest ranges, one can deduce the pure QSSR determination of $m_{s}$, to order $\alpha_{s}^{3}$ given in Table 4 and evolved to $2 \mathrm{GeV}$ in Table 6. Combining this result with the sum rules determination of $\left(m_{u}+m_{d}\right)$ in Table 1 (section (5)), one can deduce pure QSSR prediction of $m_{s} /\left(m_{u}+m_{d}\right)$ in Table 6 , which is in agreement with and an independent test of the ChPT result $24.4 \pm 1.5$ given in section (3), though less accurate.

\section{QSSR + ChPT FINAL RESULTS}

We take the average of $m_{s}$ from $m_{u}+m_{d}$ (Table 1) + the ChPT ratio and from the direct determination in Table 4. Then, we obtain the final average from QSSR+ChPT to order $\alpha_{s}^{3}$ in Table 6 . Combined with the ratios from ChPT in section 2 , this value leads to the values of $m_{u, d}$ given in Table 6. As already discussed in previous sections, the quoted error already include the systematics of the methods. The size of the error is within the expected accuracy of the sum rule results. Using Eq. 2, it is trivial to extract the value of the invariant mass $\hat{m}_{i}$. One obtains in units of $\mathrm{MeV}$ :

$\hat{m}_{u}=3.9 \pm 0.7, \quad \hat{m}_{d}=7.1 \pm 0.8$

$\hat{m}_{s}=133.3 \pm 18.8$,

where the error is larger than the corresponding running mass due to $\Lambda$ in the evolution procedure.

\section{COMPARISON WITH THE LATTICE}

8.1. Lattice approaches for/by non-experts One usually starts from the QCD action and partition function:

$Z=\int \mathcal{D} A_{\mu} \operatorname{det} M e^{\int d^{4} x\left(-\frac{1}{4} G^{\mu \nu} G_{\mu \nu}\right)}$

integrated over gauge field configurations. The fermion contributions are included into the nonlocal det $M$ term. For the analysis, one works like in the sum rule approach, with the 2-point correlator defined in previous sections, which is saturated by the intermediate states $|n\rangle\langle n|$. In this way, the two-point correlator can be expressed as:

$$
\begin{aligned}
& \sum_{x}\left\langle 0\left|J(x)(J(0))^{\dagger}\right| 0\right\rangle= \\
& \sum_{n}\langle 0|J(x)| n\rangle\left\langle n\left|(J(0))^{\dagger}\right| 0\right\rangle \frac{e^{-E_{n} t}}{2 E_{n}}
\end{aligned}
$$

where the zero momentum states $E_{n}$ tend to the masses $M_{n}$ of the resonances. In the (ideal) asymptotic limit $t \rightarrow \infty$, the exponential factor kills the effect of the different excitations, such that the lowest ground state contribution dominates. In practice, this approximation is expected to be realized when the splitting between 
the ground state and the radial excitation is large enough.

\subsection{Practical limits of the lattice}

Besides the usual statistical and finite size (about $1 \%$ if the lattice size $L \geq 3$ fermi and $m_{\pi} L \geq 6$ ), errors inherent to the lattice, which can be minimized using modern technology, there are still large uncertainties related to the uses of field theory on the lattice due to the finite values of the lattice spacing $a$ :

- The different operators mix at finite $a$.

- The discretization errors specific to each actions, which are $\mathcal{O}(a)$ for the Wilson (explicit breaking of chiral symmetry $(\chi S)$ ) and Domain wall (extra 5th dimension in order to preserve $\chi S)$ actions, $\mathcal{O}\left(a^{2}\right)$ for the staggered (reduction of quark couplings with high-momenta gluons) and $\mathcal{O}\left(a \alpha_{s}\right)$ for the Clover (inclusion of the mixed quark-gluon operator) actions. For typical values of $1 / a \approx 2 \mathrm{GeV}$, the error is $\approx 10-30 \%$, which can be reduced by computing at different values of $a$.

- The well-known quenched approximation (no inclusion of the fermion contribution $\ln$ Det $M$ ), which implies a modification of $\chi S$ with unphysical singularities for $m_{q}=0$ or practically for $m_{q} \leq m_{s} / 3$ (recall that in this approximation: $M_{\eta^{\prime}} \approx m_{\pi}=0$ (三 large $N_{c^{-}}$limit $)$), which induces an error of about $20 \%$ that can be estimated from the deviation of the predictions from the observed meson masses and couplings or/and from the choices of the mesons for setting the scale (string tension).

- The extrapolation of the results to light quark masses with the help of the meson mass dependence expected from ChPT, which for typical values $1 / a=2 \mathrm{GeV}$, and keeping $m_{\pi} L \geq 6$, one requires $L / a \geq 90$ in order to avoid finite volume effects. At present, $L / a \approx 32$ (quenched) and $L / a \approx 24$ (unquenched) far below this limit.

- The errors due to the matching of the lattice and the continuum at a typical lattice conversion scale of $2 \mathrm{GeV}$ can be minimized using the nonperturbative renormalization.

\subsection{Lattice results and estimated errors}

From the previous discussions, we consider that:

- The conservative quenched lattice errors are
Table 5

Lattice Quenched Results up to NNLO since 98.

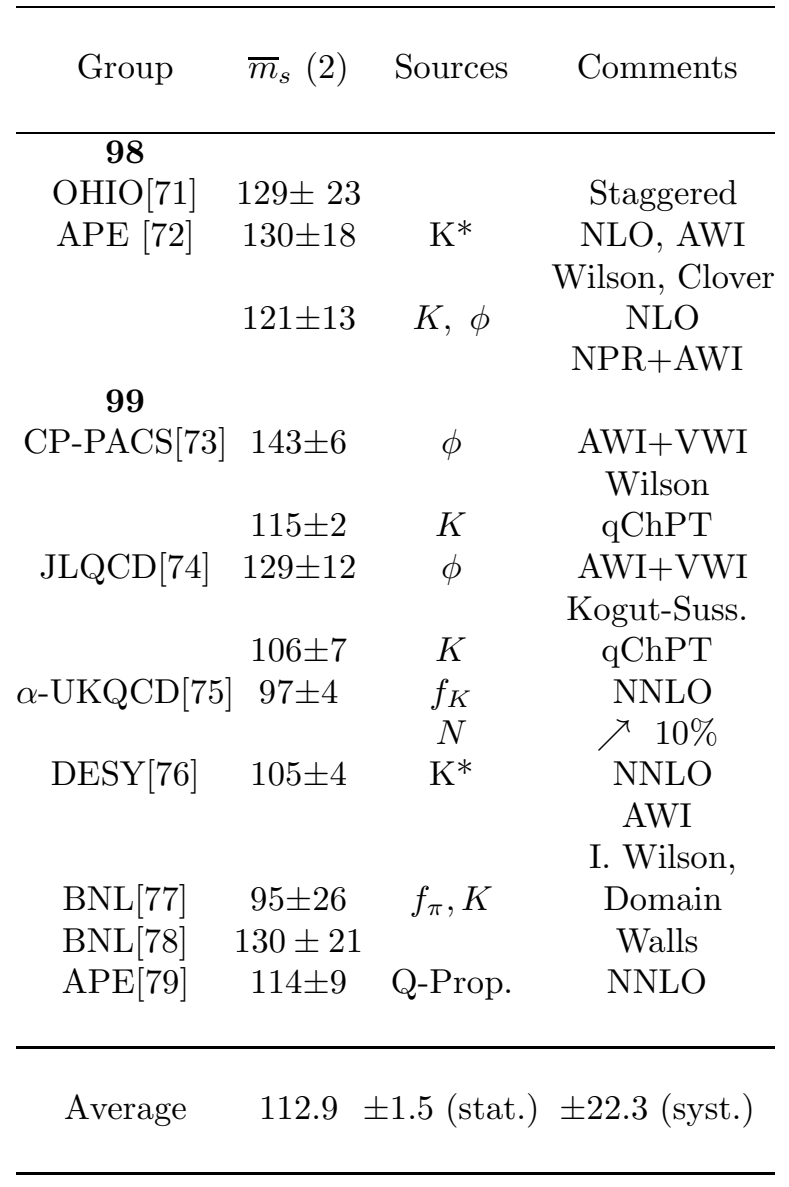

about $20 \%$.

- The extraction of $m_{u, d}$ is less reliable than $m_{s}$. Therefore, we shall only consider the value of $m_{s}$ obtained from the lattice which we shall compare with the one obtained in previous sections. Lattice results prior 98 have been already reviewed in [13]. The different results for 98 and 99 are given in Table 5 for different actions, where one can see a large spread of predictions, which with the given errors are inconsistent each others. We mainly attribute the source of this discrepancy to the underestimate of the systematic errors given there. Most of these results have been obtained using the non-perturbative renormalization [80], and Ward identities for the axial (AWI) and/or vec- 
Table 6

Summary for $\bar{m}_{u, d, s}(2)$ in $\mathrm{MeV}$

Sources $\quad \bar{m}_{s} 2 m_{s} /\left(m_{u}+m_{d}\right) \quad \bar{m}_{u}+\bar{m}_{d} \quad m_{u} / m_{d} \quad \bar{m}_{u} \quad \bar{m}_{d}$

QSSR

Table 1

Table 4

Average

$$
\begin{array}{rr}
115.8 \pm 19.7 & \Longleftarrow \mathrm{ChPT}+ \\
\mathbf{1 2 0 . 8} \pm \mathbf{1 4 . 6} & \Longleftrightarrow \mathbf{2 5 . 5} \pm \mathbf{4 . 8} \\
\mathbf{1 1 8 . 9} \pm \mathbf{1 2 . 2} & +\mathrm{ChPT} \Longrightarrow
\end{array}
$$

$\mathbf{9 . 5} \pm \mathbf{1 . 4}+\mathrm{ChPT} \Longrightarrow 3.4 \pm 0.66 .1 \pm 0.8$

$9.8 \pm 1.2+\mathrm{ChPT} \Longrightarrow 3.5 \pm 0.46 .3 \pm 0.8$

\section{LATTICE}

Table 5: quenched

$$
112.9 \pm 22.4 *
$$

less reliable

$n_{f}=2$ dynamical [83]

$97 \pm 11 \sqrt{2}$ *

less reliable

\begin{tabular}{ll} 
GRAND AVERAGE $\quad \mathbf{1 1 0 . 9} \pm \mathbf{8 . 8}+\mathrm{ChPT} \Longrightarrow$ & $9.1 \pm 0.9+\mathrm{ChPT} \Longrightarrow 3.2 \pm 0.35 .9 \pm 0.5$ \\
$\begin{array}{l}\text { QSSR BOUNDS } \quad \mathbf{9 0} \leq \boldsymbol{\cdots} \leq \mathbf{1 6 8} \\
\text { Tables 2 and } 3\end{array}$ & $\mathbf{7} \leq \mathbf{\ldots} \leq \mathbf{1 2}$ \\
\hline
\end{tabular}

* Our conservative error estimate

tor (VWI) currents, and constraints from ChPT in the extrapolation procedure. There is also a systematic discrepancy between the results from the kaon and $\phi, K^{*}$ or the nucleon masses, which might be some indications of the quenching errors, while e.g. the splitting of the $K-K^{*}$ is no longer resolved. As one can see from Table 5, the quenched lattice predictions are in the range:

$\bar{m}_{s}(2) \simeq(69-181) \mathrm{MeV}$,

where part of this range is already excluded by the bounds given in Tables 2 and 3. Instead, one can also quote (to be taken carefully) the naïve average given in Table 5 at NNLO 7 , where we have added our guessed $20 \%$ estimate of the lattice systematic errors based on the previous comments. At this approximation, where a comparison with the previous results from QCD spectral sum rules is meaningful, one can notice a surprisingly good agreement.

Some attempts to put dynamical fermions have

\footnotetext{
${ }^{7}$ However, as discussed in 79 , the jnclusion of the higher order $\alpha_{s}$ corrections obtained in 81] in the conversion of the lattice to the continuum results tends to decrease slightly the value of $m_{s}$ by about $3 \%$.
}

been done in 82 and more recently with 2 flavours in 83. In 83], some of the problems encountered in the quenched approximation (discrepancy between the $\phi$ and $K$ results,...) seems to be resolved. Though promising, the approach is not enough mature for the different systematics to be fully under control. We quote in Table 6 this result adopting a more conservative error than the original one.

\section{SUMMARY}

We have reviewed the different determinations of light quark masses from $\mathrm{ChPT}$, QCD spectral sum rules (QSSR), $e^{+} e^{-}$and $\tau$-decay data, and compared the one of the strange quark mass with the recent lattice results:

- The sum of light quark masses $m_{u}+m_{d}$ to order $\alpha_{s}^{3}$ from different QSSR analysis is given in Table 1 and the resulting average value.

- Lower (resp. upper) bounds based on the positivity and analyticity properties of the spectral functions are given in Table 2 (resp. Table 3). - Different direct determinations of the strange quark mass to order $\alpha_{s}^{3}$ are compiled in Table 4 .

- Combined results from these four methods lead 
to the final average given in Table 6 to order $\alpha_{s}^{3}$, where the errors are typically the $10 \%$ systematics of the QSSR approach. An eventual failure of this result should signal a new phenomena not accounted for in the OPE discussed in this paper.

- We have compared this final result with the recent (after 98) lattice determinations (Table 5) which belong in the range given by Eq. 27 and which lead to the average in Table 5 .

- Within the present uncertainties of various approaches, we consider that there is a good agreement between the previous sum rule and lattice results. Attempting to give the final QCD value, we average the different results in Table 6 , and deduce the $Q C D$ Grand Average given in this table, to be used carefully.

- However, we expect that a future high-precision measurement of the light quark masses will be difficult to reach due to the systematic errors inherent to each methods, which, often, different authors do not include into their results !

- Finally, one should remind that, in the phenomenological analysis, one should use the value of $m_{s}$ into the expression of any hadronic matrix elements or/and observables which are known at the same level of approximation. This consistency condition is not often respected in the literature.

\section{APPLICATION TO $\epsilon^{\prime} / \epsilon$}

One of the most fashionable applications of the previous result is the one to the $\mathrm{CP}$ violating parameters $\epsilon^{\prime} / \epsilon$, where $\epsilon^{\prime}$ is related to $A\left[K_{L} \rightarrow\right.$ $\left.(\pi \pi)_{I=2}\right] / A\left[K_{S} \rightarrow(\pi \pi)_{I=0}\right]$ and characterizes the (direct) CP-violation in the decay amplitude of $K \rightarrow \pi \pi ; \epsilon=A\left[K_{L} \rightarrow(\pi \pi)_{I=0}\right] / A\left[K_{S} \rightarrow\right.$ $\left.(\pi \pi)_{I=0}\right]$ is the (indirect) CP-violation from $K^{0}$ $\bar{K}^{0}$ mixing. It is known [5] that the dominant effects in the analysis of $K_{L, S} \rightarrow(\pi \pi)_{I=0,2}$ amplitudes are due to the QCD and electroweak penguin operators:

$$
\begin{aligned}
Q_{6} & \equiv\left(\bar{s}_{\alpha} d_{\beta}\right)_{V-A} \sum_{u, d, s}\left(\bar{\psi}_{\alpha} \psi_{\beta}\right)_{V+A} \\
& \approx B_{6}^{1 / 2} / m_{s}^{2}+\mathcal{O}(1 / N) \\
Q_{8} & \equiv \frac{3}{2}\left(\bar{s}_{\alpha} d_{\beta}\right)_{V-A} \sum_{u, d, s}\left(e_{\psi} \bar{\psi}_{\alpha} \psi_{\beta}\right)_{V+A}
\end{aligned}
$$

$$
\approx B_{8}^{3 / 2} / m_{s}^{2}+\mathcal{O}(1 / N)
$$

For $M_{t} \simeq 165 \mathrm{GeV}$ and $\Lambda_{4} \simeq 340 \mathrm{MeV}$, the simplified SM prediction without (resp. with) the inclusion of final-state interaction effects 84, is [5]:

$$
\begin{aligned}
\frac{\epsilon^{\prime}}{\epsilon} \approx & 9.75(\text { resp. } 15.34) \operatorname{Im} \lambda_{t}\left[\frac{110 \mathrm{MeV}}{\bar{m}_{s}(2)}\right]^{2} \\
& \times\left[B_{6}^{1 / 2}-0.54\left(\text { resp.0.32) } \mathrm{B}_{8}^{3 / 2}\right] .\right.
\end{aligned}
$$

where $\lambda_{t}=V_{t d} V_{t s}^{*}$ is expressed in terms of the CKM matrix elements. Using $\operatorname{Im} \lambda_{t} \simeq(1.34 \pm$ $0.30) \times 10^{-4}[5,85$, and the measured value [6] $\left(\epsilon^{\prime} / \epsilon\right)_{\text {exp }} \simeq(21.4 \pm 4.0) \times 10^{-4}$, one can deduce, from the average value and the lower bound of $m_{s}$ in Table 6 :

$$
\begin{aligned}
B_{6}^{1 / 2}-0.54(0.32) B_{8}^{3 / 2} & \simeq 1.6 \pm 0.4(1.1 \pm 0.3) \\
& \geq 1.1 \pm 0.2(0.7 \pm 0.1)
\end{aligned}
$$

which signals a violation of about $1 \sim 3 \sigma$ for the leading $1 / N$ vacuum saturation prediction ?: $B_{6}^{1 / 2} \approx B_{8}^{3 / 2} \approx 1$. This result and the final-state interaction effects should be tested using more accurate non-perturbative calculations. It is only after performing these tests that one can make a sharper conclusion on the SM prediction of the $C P$-violation 10 .

\section{REFERENCES}

1. S. Weinberg, in a Festschrift for I.I. Rabi, ed. L. Motz, Trans. New York Acad. Sci. Ser. II 38 (1977) 185; P. Langacker and H. Pagels, Phys. Rev. D19 (1979) 2070; P. Langacker, Phys. Rev. D20 (1979) 2983.

2. J. Gasser and H. Leutwyler, Phys. Reports 87 (1982) 79 and references therein.

3. S. Weinberg, Physica A96 (1979) 327; J. Gasser and H. Leutwyler, Ann. Phys. 158 (1984) 142; Nucl. Phys. B250 (1985) 465;

\footnotetext{
${ }^{8}$ This is an improved estimate of the one in 65

${ }^{9}$ It has been noticed from various QSSR analysis 12 that the four-quark vacuum condensate also deviates from the vacuum saturation by about a factor 2 to 3 .

${ }^{10} \mathrm{~A}$ direct analysis can avoid the $m_{s}$-dependence 86$]$.
} 
E. de Rafael, hep-ph/9502254 and references therein; A. Pich, hep-ph/9502366; A. Manohar, hep-ph/9607484; J. Gasser, these proceedings; E. de Rafael, M. Knecht and S. Peris, these proceedings.

4. See e.g. H. Fritzsch, Nucl. Phys. (Proc. Sup.) B64 (1997) 271

5. S. Bosch et al., hep-ph/9904408; S. Bertolini et al., hep-ph/9802405; M. Fabbrichesi, these proceedings; U. Nierste, these proceedings; E. de Rafael, in Ref. [3]; M. Jamin, hepph/9911390.

6. B. Hsiung for the $\mathrm{kTeV}$ collaboration (these proceedings); ibid, B. Peyaud for the NA48 collaboration.

7. R. Coquereaux, Phys. Rev. D23 (1981) 1365;

R. Tarrach, Nucl. Phys. B183 (1981) 384.

8. S. Narison, Phys. Lett. B197 (1987) 405; B216 (1989) 191.

9. E.G. Floratos, S. Narison and E. de Rafael, Nucl. Phys. B155 (1979) 115.

10. PDG96, R.M. Barnett et al., Phys. Rev. D54 (1996) 1; PDG97, L. Montanet et al., Phys. Rev. D54 (1997) 1; PDG98, C. Caso et al., Eur. Phys. J C3 (1998) 1.

11. M.A. Shifman, A.I. Vainshtein and V.I. Zakharov, Nucl. Phys. B147 (1979) 385, 448.

12. For a review and references to original works, see e.g., S. Narison, QCD spectral sum rules, Lecture Notes in Physics, Vol 26 (1989) ed. World Scientific; Acta Phys. Pol. 26 (1995) 687; Recent Progress in QCD spectral sum rules (to appear).

13. For reviews see e.g.: C. Allton, Nucl. Phys. (Proc. Sup.) B64 242 (1998); R. Gupta, hep-ph/9801412 (1998); R.D. Kenway, heplat/9810054 (1998); V. Lubicz, Nucl. Phys. (Proc. Sup.) B74 (1999) 249; G. Martinelli, Hadron 99; R. Petronzio, Lattice 99.

14. ALEPH collaboration : M. Davier, Nucl. Phys. (Proc. Sup.) C55 (1997) 395; S. Chen, Nucl. Phys. (Proc. Sup.) B64 (1998) 265; E. Tourenefier, these proceedings.

15. T. van Ritbergen, J.A.M. Vermaseren and S.A. Larin, Phys. Lett. 400 (1997) 305; B405 (1997) 327; K.G. Chetyrkin, Phys. Lett. B404 (1997) 161.

16. M. Gell-Mann, R.J. Oakes and B. Renner,
Phys. Rev. 175 (1968) 2195.

17. H. Leutwyler, hep-ph/9609467 (1996) and references therein.

18. M. Knecht and J. Stern, Nucl. Phys. (Proc. Sup.) B, C39 (1995) 249; B. Moussallam, these proceedings.

19. R. Dashen, Phys. Rev. 183 (1969) 1245.

20. D. Kaplan and A. Manohar, Phys. Rev. Lett. 56 (1986) 2004.

21. For a review, see e.g. V.A. Zakharov, Nucl. Phys. (Proc. Sup.) B74 (1999) 392; ibid, these proceedings; F.V. Gubarev, M.I. Polikarpov and V.I. Zakharov, hep-th/9812030.

22. G.S. Bali, hep-ph/9905387.

23. S. Narison, Phys. Lett. B300 (1993) 293; ibid B361 (1995) 121.

24. K. Chetyrkin, S. Narison and V.I. Zakharov, Nucl. Phys. B550 (1999) 353.

25. See e.g.: M. Teper, hep-lat/9711011; C. Michael, hep-ph/9710249.

26. Y. Chung et al.Z. Phys. C25 (1984) 151; H.G. Dosch, M. Jamin and S. Narison, Phys. Lett. B220 (1989) 251.

27. S. Narison, Phys. Lett. B387 (1996) 162.

28. J.S. Bell and R.A. Bertlmann, Nucl. Phys. B177 (1981) 218; B187 (1981) 285; R.A. Bertlmann, Acta Phys. Austr. 53 (1981) 305.

29. F.J. Yndurain, hep-ph/9903457.

30. M. D'Elia, A. Di Giacomo and E. Meggiolaro, Phys. Lett. B408 (1997) 315.

31. B.L. Ioffe, Nucl. Phys. B188 (1981) 317 , B191 (1981) 591;

32. S. Narison, Phys. Lett. B210 (1988) 238.

33. V.A. Novikov et al., Nucl. Phys. B191 (1981) 301.

34. S. Narison and E. de Rafael, Phys. Lett. B103 (1981) 57.

35. E. Braaten, S. Narison and A. Pich, Nucl. Phys. B373 (1992) 581; S. Narison and A. Pich, Phys. Lett. B304 (1993) 359.

36. F. Le Diberder and A. Pich, Phys. Lett. B286 (1992) 147 and B289 (1992) 165.

37. S. Narison, Phys. Lett. B358 (1995) 113.

38. S.J. Brodsky, J.R. Pelaez and N. Toumbas, hep-ph/9810424.

39. R.A. Bertlmann, G. Launer and E. de Rafael, Nucl. Phys. B250 (1985) 61; R.A. Bertlmann et al., Z. Phys. C39 (1988) 231. 
40. K. Chetyrkin, N. V. Krasnikov and A. N. Tavkhelidze, Phys. Lett. B76 (1978) 83; N. V. Krasnikov, A. A. Pivovarov and A. N. Tavkhelidze, Z. Phys. C19 (1983) 301.

41. S.G. Gorishny, A.L. Kataev and S.A. Larin, Phys. Lett. B135 (1984) 457.

42. C. Becchi, S. Narison, E de Rafael and F.J. Yndurain, Z. Phys. C8 (1981) 335.

43. E. de Rafael, Lectures given at the Dubrovnik's school (1983).

44. S. Narison, Rev. Nuov. Cim. Vol 10 (1987) 1; Phys. Lett. B216 (1989) 191.

45. C.A. Dominguez and E de Rafael, Ann. Phys. 174 (1987) 372.

46. J. Bijnens, J. Prades and E. de Rafael, Phys. Lett. B348 (1995) 226.

47. J. Prades, Nucl. Phys. (Proc. Sup.) B64 (1998) 253 and private communication.

48. T. Bhattacharya, R. Gupta and K. Maltman, Phys. Rev. D57 (1998) 5455.

49. L. Lellouch, E. de Rafael and J. Taron, Phys. Lett. B414 (1997) 195; E. de Rafael, Nucl. Phys. (Proc. Sup.) B64 258 (1998).

50. F.J. Yndurain, Nucl. Phys. B517 (1998) 324.

51. K.G. Chetyrkin, D. Pirjol and K. Schilcher, Phys. Lett. B404 (1997) 337.

52. H.G. Dosch and S. Narison, Phys. Lett. B417 (1998) 173.

53. L. Giusti et al., hep-lat/9807014.

54. S. Narison, N. Paver, D. Treleani and E. de Rafael, Nucl. Phys. B212 (1983) 365.

55. A. Bramon and S. Narison, Mod. Phys. Lett. A4 (1989) 1113; L. Montanet, these proceedings.

56. S. Narison and G. Veneziano, Int. J. Mod. Phys. A4, 11 (1989) 2751.

57. S. Narison, Nucl. Phys. B509 (1998) 312; Nucl. Phys. (Proc. Sup.) B64 (1998) 210; Hadron 99, hep-ph/9909470; P. Minkowski, these proceedings.

58. S. Bethke, Nucl. Phys. (Proc. Suppl.) B, C39 (1995); ibid, B, A54 (1997); I. Hinchliffe, Meeting of the American Physical Society, Albuquerque, 1994; M. Schmelling, ICHEP96, Varsaw,1996.

59. C. A. Dominguez, L. Pirovano and K. Schilcher, Nucl. Phys. (Proc. Sup.) B74 (1999) 313 and references therein.
60. K.G. Chetyrkin, C.A. Dominguez, D. Pirjol and K. Schilcher, Phys. Rev. D51 (1995) 5090.

61. M. Jamin and M. Munz, Z. Phys. C66 (1995) 633.

62. P. Colangelo, F. De Fazio, G. Nardulli and N. Paver, Phys. Lett. B408 (1997) 340.

63. M. Jamin, Nucl. Phys. (Proc. Sup.) B64 (1998) 250.

64. K. Maltman, Phys. Lett. B462 (1999) 195.

65. S. Narison, Phys. Lett. B466 (1999) 345.

66. T. Das, V.S. Mathur and S. Okubo, Phys. Rev. Lett. 19 (1967) 470.

67. K. Maltman, Phys. Lett. B440 (1998) 367.

68. K.G. Chetyrkin, J.H. Kühn and A.A. Pivovarov, Nucl. Phys. B533 (1998) 473.

69. J. Prades and A. Pich, Nucl. Phys. (Proc. Sup.) B74 (1999) 309.

70. J. Prades and A. Pich, these proceedings.

71. D. Pekurovsky and G. Kilcup , hep - lat / 9809115.

72. V. Gimenez et al., hep-lat/9809037.

73. S. Aoki et al., hep-lat/9904012.

74. S. Aoki et al., hep-lat/9901019.

75. J. Garden et al., hep-lat/9906013.

76. M. Göckeler et al., hep-lat/9908005.

77. T. Blum, A. Soni and M. Wingate, heplat/9902016; M. Wingate, these proceedings.

78. M. Wingate, these proceedings.

79. D. Becirevic et al., hep-lat/9909082.

80. G. Martinelli et al., Nucl. Phys. B445 (1995) 81.

81. K. Chetyrkin, these proceedings.

82. N. Eicker et al., hep-lat/9806027.

83. CP-PACS: R. Burkhalter and T. Kaneko (private communication).

84. E. Pallante and A. Pich, hep-ph/9911233; see however, A.J. Buras et al., hep-ph/0002116.

85. A. Ali and D. London, Eur. Phys. J. C9 (1999) 687.

86. L. Giusti, this proceedings.

\section{QUESTIONS AND DISCUSSIONS}

Lively general discussions, many questions and some comments have followed this talk, As they have been also addressed to the previous talks in this session, they have not been reported here. 